\title{
Corela
}

Cognition, représentation, langage

HS-31 | 2020

Métalinguistiques.

\section{Hétérogénéité et valeur du discours littéraire}

\section{Stéphane Bikialo}

\section{(2) OpenEdition}

Journals

Édition électronique

URL : http://journals.openedition.org/corela/11528

DOI : 10.4000/corela. 11528

ISSN : 1638-573X

Éditeur

Cercle linguistique du Centre et de l'Ouest - CerLICO

Référence électronique

Stéphane Bikialo, «Hétérogénéité et valeur du discours littéraire », Corela [En ligne], HS-31 | 2020, mis en ligne le 02 juillet 2020, consulté le 03 juillet 2020. URL : http://journals.openedition.org/corela/ 11528 ; DOl : https://doi.org/10.4000/corela.11528

Ce document a été généré automatiquement le 3 juillet 2020.

\section{(c) (i) (2) (2)}

Corela - cognition, représentation, langage est mis à disposition selon les termes de la licence Creative Commons Attribution - Pas d'Utilisation Commerciale - Partage dans les Mêmes Conditions 4.0 International. 


\title{
Hétérogénéité et valeur du discours littéraire
}

\author{
Stéphane Bikialo
}

1 En quoi le métalinguistique et l'hétérogénéité interviennent-ils dans la valeur esthétique d'un discours littéraire? Par valeur, je n'entends pas une supposée spécificité du discours littéraire - cette littérarité, qui me semble être une notion périmée, voire une fiction ${ }^{1}$ - mais une dimension qualitative. La valeur est ce qui fait qu'un discours acquière une singularité, artistique (discours littéraire) ou non (discours ordinaire, discours politique, etc.)? Dans le cas du discours littéraire, cette valeur repose sur un grand nombre d'instances de légitimation internes (les genres littéraires notamment) et externes (pairs, éditeurs, critiques, journalistes, lecteurs, jurys de prix littéraires, programmateurs de festivals, etc.). Par ailleurs, cette singularisation s'inscrit au sein d'une œuvre, et la valeur de cette œuvre reposera en grande partie sur sa capacité à surprendre, à ne pas être attendue ${ }^{2}$, à changer quelque chose au lecteur (singulier ou collectif) qui la reçoit dans ses modes de représentation (de soi, des autres, du monde, de la langue, du monde). Dans la mesure où la littérature est l'art du langage, l'attention à la matérialité discursive, voire à la manière dont le signe s'interpose, va être fondamentale dans la détermination de cette valeur. Et c'est là que l'hétérogène et le métalinguistique interviennent.

2 Rappelons avec J. Authier-Revuz que si l'hétérogène est constitutif de tout énoncé, il peut être latent, non représenté (dans énoncés apparemment monologiques) ou manifeste voire montré :

a. il y a de l'hétérogène constitutif de l'énonciation qui est présent, à l'œuvre, de façon permanente, mais non directement observable ;

b. il y a de l'hétérogène manifeste, sur le fil, y produisant des ruptures observables.

Cet hétérogène est de deux sortes :

- b1. de l'hétérogène émergeant de façon brute, simplement manifeste.

- b2. de l'hétérogène émergeant sous les espèces de sa représentation par le sujet parlant lui-même, hétérogène montré (1991: 143). ${ }^{3}$

3 L'hypothèse ici travaillée est que la valeur d'un discours littéraire s'évaluerait - en partie (ou en particulier) - à partir de la forme de négociation que l'œuvre effectue 
avec l'hétérogénéité constitutive et avec les formes de représentation de cette hétérogénéité ${ }^{4}$ cette négociation permettant de distinguer les discours inquiets (ordinaires, littéraires...), et les discours sans inquiétude, homogènes, uniformes. J. Authier-Revuz évoque ainsi les énonciateurs sourds, à qui il «n'arrive rien » dans leur dire (2007: 122) et ceux à l'inverse, « énonciateurs sur le qui-vive », à l'écoute, jusqu'aux positions extrêmes consistant à " céder l'initiative aux mots ", et elle ajoute, à travers une analogie entre la démarche du linguiste et celle de l'écrivain :

Si le langage, le dire, les mots sont de l'ordre de «ce qui va de soi », de ce qu'on utilise sans y penser, qu'on «traverse » sans le percevoir dans son mouvement vers les choses (qu'on nomme) et les autres (à qui on s'adresse), si on est « installé » dans une sorte de neutralité tranquille dans le langage... je ne pense pas qu'on devienne linguiste ou écrivain. [...] Certes, à l'évidence, ce rapport distancié au langage se résout sur des modes opposés dans ces deux pratiques : pour l'un, le linguiste, en occupant une position de surplomb métalinguistique par rapport au langage et à la langue [...] ; pour l'autre, le questionnement du sujet du langage se résout en une pratique du langage qui est à elle-même sa propre fin, incorporant, de façon singulière pour chaque sujet, ce questionnement dans une dimension réflexive interne travaillant l'écriture. (Authier-Revuz 2007: 116)

4 Afin d'étayer cette hypothèse, je m'appuierai d'abord sur les approches qui ont vu dans le métalinguistique un trait voire un critère de la littérarité dans les années 1950-1970. Puis je m'attacherai à décrire quelques manifestations de cette mise en jeu de l'hétérogénéité manifeste ou représentée et du métalinguistique dans des œuvres afin de montrer que leur densité est un des éléments de la valeur de l'œuvre littéraire.

\section{Le métalinguistique comme trait ou critère de la littérarité}

\subsection{Un trait synchronique}

5 Il se pourrait que le métalinguistique soit un trait fondamental d'une certaine période historique (l'avant-garde dans la mouvance du structuralisme) ou de tels genres littéraires (la poésie) : M. Foucault écrit ainsi qu'à partir de la fin du XIX ${ }^{\mathrm{e}}$ siècle la

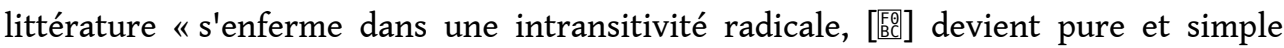
manifestation d'un langage qui n'a pour loi que d'affirmer 膯 contre tous les autres discours 閲 son existence escarpée ; elle n'a plus alors qu'à se recourber dans un perpétuel retour sur soi comme subjectivité écrivante » (1966: 313). Et il évoque, dans «La pensée du dehors » le remplacement moderne du je pense par un je parle:

On a l'habitude de croire que la littérature moderne se caractérise par un redoublement qui lui permettrait de se désigner elle-même; en cette autoréférence, elle aurait trouvé le moyen à la fois de s'intérioriser à l'extrême (de n'être plus que l'énoncé d'elle-même) et de se manifester dans le signe scintillant de sa lointaine existence. En fait, l'événement qui a fait naître ce qu'au sens strict on entend par « littérature » n'est de l'ordre de l'intériorisation que pour un regard de surface; il s'agit beaucoup plus d'un passage au «dehors »: le langage échappe au mode d'être du discours - c'est-à-dire à la dynastie de la représentation -, et la parole littéraire se développe à partir d'elle-même, formant un réseau dont chaque point, distinct des autres, à distance même des plus voisins, est situé par rapport à tous dans un espace qui à la fois les loge et les sépare. La littérature, ce n'est pas le langage se rapprochant de soi jusqu'au point de sa brûlante manifestation, c'est le langage se mettant au plus loin de lui-même. [...] Le « sujet » de la littérature (ce qui 
parle en elle et ce dont elle parle), ce ne serait pas tellement le langage en sa positivité, que le vide où il trouve son espace quand il s'énonce dans la nudité du

« je parle ». (1966a: 547-548)

6 Dire que (une part de) la littérature contemporaine se caractérise comme un je parle, c'est insister sur l'importance du méta- et de l'épilinguistique ${ }^{5}$ comme objet même de l'histoire racontée. D. Rabaté, décrivant l'émergence de la notion de " récit » à la fin du $\mathrm{XIX}^{\mathrm{e}}$ et au début du XX $\mathrm{XX}^{\mathrm{e}}$ siècle (Monsieur Teste de Valéry, Paludes de Gide, Nadja de Breton), puis dans les années 1950 avec Camus, Des Forêts et Beckett, souligne que le récit - par opposition au roman et au poème - serait un espace de réflexivité du sujet de l'écriture, où le sujet se construit tout en se dérobant : « espace toujours contesté de se saisir par soi-même. Espace, si l'on veut, du JE-ME. [...] De la voix, il a la réflexivité douloureuse : je m'entends parler. $»^{6}$

7 À cette analyse correspond la mise au jour, par Gilles Philippe (2002) d'un «moment grammatical » de la littérature (1890-1940), la grammaticalisation des discours sur la littérature, provoquant «l'avènement d'un concept nouveau, qui restera innommé pendant toute la période concernée, celui de "littérarité" » (2002: 13). Par la suite, pour Barthes, à partir du Degré zéro de l'écriture (1953), comme le résume Gilles Philippe, «le propre de la littérarité, c'est l'autonymie : un texte est littéraire à partir de l'instant où il donne des signes de son appartenance à la littérature » (2002: 208). Cette hypothèse de la dimension méta- comme trait de la modernité littéraire est à nuancer non seulement en prenant en compte un certain nombre d'œuvres antérieures (ou en soulignant que la modernité n'est pas liée à la date de publication) - comme celles de Rabelais, Montaigne, Marivaux, Flaubert, Balzac, Stendhal... - mais aussi théoriquement - en envisageant cette dimension méta- comme un trait constitutif du discours littéraire, avec des variations selon les pratiques et périodes.

\subsection{Littérature et mode du comme je dis}

C'est ainsi, au-delà de l'historicité, comme caractérisation générale et théorique du fait littéraire, que le métalinguistique peut intervenir. J. Rey-Debove (1978), dans le cadre de sa réflexion sur la « connotation autonymique $»^{7}$, a montré que celle-ci met en jeu différents modes $d u$ dire autonymes qui peuvent être explicites (avec commentaire métalinguistique) ou non, et à connotation continue ou sporadique (Rey-Debove 1978(1997): 267-268). Elle distingue quatre modes essentiels :

Comme un autre dit (il, $\mathrm{tu})$

Comme certains disent (ils, vous)

Comme tout le monde dit (on)

Comme je dis (je).

Cette réflexion débouche sur une caractérisation de la littérarité comme mode du comme je dis $^{8}$ :

Il y a deux façons de signifier un signe déterminé, soit d'être un signe autonyme, soit d'être un signe à connotation autonymique. La première hypothèse est absurde, car elle ferait du texte littéraire le nom d'un texte [...] ; le texte dénoterait un macrosigne et n'informerait que sur lui, par iconicité. La seconde hypothèse semble convenir à la situation ; la littérature parle du monde, mais signifie des signes déterminés comme connotés de façon continue, sur le mode du Comme je dis. La forme apparaît non seulement en tant que signifiant, mais comme signifié connotatif [...]. Cette interprétation est liée à l'opacité du texte littéraire. Dans la communication courante, le discours est transparent, le signe n'est jamais perçu en 
tant que tel, sauf lorsqu'il est inconnu [...]. Dans le texte littéraire, et spécialement le texte poétique, où l'expression personnelle (Comme je dis) se donne libre cours, construisant son propre code, le discours s'opacifie et impose ses signes au décodeur (1978 (1997): 288).

10 En littérature, le mode du comme je dis serait donc à la fois implicite et continu. Cette hypothèse d'une opacification généralisée, d'un texte "tout entier entre guillemets » selon l'expression de M. Bakhtine, rend parfaitement compte du fait que tout texte littéraire parle à la fois du monde et des mots qu'il emploie pour en parler. L'interprétation autonymique du texte littéraire permet à la fois de comprendre sa double référence - au monde et aux mots.

11 J. Rey-Debove liste un certain nombre de critères qui témoigneraient de, ou permettraient de décrire, cette dimension de connotation autonymique à l'œuvre dans tout discours littéraire : l'accent mis sur le signifiant ${ }^{9}$ et plus précisément l'insertion du signifiant dans le signifié - qui implique un blocage de la synonymie -, la dimension connotative, la présence abondante de métaphores, la projection de l'axe paradigmatique sur l'axe syntagmatique ${ }^{10}$.

12 Avant de prendre au mot et de chercher à exemplifier cette hypothèse d'une opacification généralisée pour caractériser le discours littéraire en évoquant un certain nombre d'œuvres littéraires, je formulerais deux nuances ou réserves :

13 - La formulation en comme je dis suppose une maîtrise du sujet qui ne convient que très partiellement au texte littéraire, tendant plutôt vers un sujet effet (Authier-Revuz, 1995/2012: 77-78) ; suivant J. Authier-Revuz, je propose de reformuler ce comme je dis en je dis $X^{\prime}$ (1995/2012: 107-160) où X' est un signe en modalisation autonymique et non seulement autonyme ; tout autant implicite, cette formule abstraite du je dis $X^{\prime}$ permet de rendre compte d'une sorte de modalisation autonymique implicite et permanente, d'une opacification généralisée, ainsi que de l'omniprésence subjective, sans la maîtrise absolue du comme je dis. Cette forme peut ainsi passer par différents modes du dire, être explicitée de différentes manières, comme l'a montré J. Authier-Revuz (2000: 231) dans son travail sur l'allusion au sujet du comme on dit implicite.

14 - À la différence de J. Rey-Debove, qui oppose clairement littérature et discours ordinaire (1971: 93 et 1978(1997): 288), on peut estimer qu'«il n'y a pas de rupture entre dire "ordinaire" et écriture, enracinés dans une même expérience - humaine - de langage ", qu'il y a à l'inverse une « unité du dire spontané et de l'écriture littéraire la plus travaillée : dans tous les cas est en jeu une même matérialité de la langue et un même enjeu subjectif essentiel » (Authier-Revuz 2007: 116).

À ce qui est le fait de tout dire - que ses arrêts-sur-mots révèlent quelque chose d'un rapport générique, situationnel, personnel sur le langage - l'écriture confère une forme par ce travail de et sur le langage qui lui est propre, à même d'atteindre des positions énonciatives extrêmes, risquées, paradoxales dans le rapport au langage qui s'y accomplit (id.: 113)

On peut estimer qu'il y a un travail, une «sublimation » comme le propose M. Aquien (1989) qui travaille sur le signifiant et la manière dont l'écriture littéraire opère sur deux versants (manière de souligner l'hétérogénéité). On peut aussi mettre l'accent sur la « cohérence » (Rannoux ici-même) ou la densité au sein d'une œuvre : le travail de la forme, du matériau crée une densité, une condensation au sein de l'espace de l'œuvre ${ }^{11}$. Cette densité est liée à l'« arrêt sur mots » (Authier-Revuz 2007) qui se manifeste par une importance des non-coïncidences énonciatives, menant à des «positions énonciatives extrêmes, risquées, paradoxales ${ }^{12}$. Cette densité crée une forme 
d'« opacification généralisée » de l'œuvre littéraire. Si cette densité peut distinguer le discours littéraire du discours ordinaire ${ }^{13}$, il y a bien là une différence qui n'est pas de nature mais qui touche à la valeur. Cette densité peut se manifester dans la forte présence de telle ou telle forme de non-coïncidence comme le montre J. Authier-Revuz (1995) au sujet de la non-coïncidence du discours à lui-même chez Flaubert (1995(2012): 455-463) ou de la non-coïncidence entre les mots et les choses chez Quignard, Sarraute, Simon, Combet (1995(2012): 636-649) ; la densité peut aussi apparaitre dans le cumul des non-coïncidences dans certains poèmes de Bernard Noël (Bikialo 2012).

Cette opacification généralisée est sensible à travers le blocage de la synonymie - un des traits de la connotation modalisation autonymique; ce blocage de la synonymie intervient de manière privilégiée en poésie (ou dans les slogans publicitaires) où un mot ne peut pas être mis pour un autre (ne serait-ce qu'en raison des associations à la rime, ou des autres formes d'assonances et d'allitérations), mais également dans la prose. Prenons un extrait de La Modéliste de R. Detambel :

Sur la moquette claire, laine mouillée de taches de fraîcheur en triple auréole parfaitement concentrique, chaque aiguillée se détache et se ramifie avec une prestigieuse netteté. Une bobine bleue a roulé, s'est défaite, comme sous la griffe attentive d'une chatte. La boîte à couture, copie d'une antique malle corsaire, vernie, s'adosse à la bibliothèque. Coline a disposé, contre les reliures de l'encyclopédie, toute une panoplie de bobines vides; en minces colonnettes les a empilées (Julliard, 1990, p. 5)

Dans ce roman, tous les mots sont féminins et cette contrainte - oulipienne - de genre, ce lipogramme du masculin, bloque toute synonymie. Dans La Liseuse (P.O.L, 2012), Paul Fournel narre l'histoire d'un éditeur, qui va découvrir la lecture électronique, par le biais d'une liseuse et envisage les conséquences sur le contenu du texte de cette séparation du texte et du contenant; et pour interroger cette pluralité des supports, Fournel fait de ce roman une sextine, c'est-à-dire une forme fixe, un roman écrit « au signe près $»^{14}$, où chaque première strophe comprend 7500 signes (dont les blancs) puis 6500 , puis 5500. Dans ces deux œuvres, l'opacification est totale, tout en étant implicite, ou en tout cas non immédiatement perceptible à la lecture.

\section{Hétérogénéités contemporaines}

18 L'hétérogénéité est donc constitutive de tout discours, notamment sous les formes des dialogismes (interlocutif, interdiscursif, auto-dialogique) ou des non-coïncidences (des mots et des choses et des mots à eux-mêmes, en plus des deux formes de noncoïncidences dialogiques). Dans ces formes d'hétérogénéité dialogiques ou non, le méta- (-linguistique, -discursif, -énonciatif) est central, voire à la source de l'hétérogénéité. Je me limiterai à des œuvres où l'hétérogénéité est représentée, et pas seulement manifeste. Un certain nombre d'œuvres modernes et contemporaines font explicitement du langage le sujet même de l'œuvre, dont la dimension métalinguistique est donc au cœur du projet littéraire.

\subsection{Le langage objet du dire}

19 Dans certaines œuvres, le langage est le thème, ce dont on parle; le langage n'est pas seulement "rappelé », il est l'objet du dire; ces œuvres sont donc thématiquement métalinguistiques. Ainsi Le Bavard (1946) de Louis-René Des Forêts met-il en scène un 
personnage éponyme qui effectue et commente une « crise de bavardage » (p. 140), le récit n'ayant d'autre fonction que de lui permettre de "parler [...] de [s]on besoin de parler » (p. 145) ; les commentaires méta-énonciatifs abondent dans ce récit. L'œuvre de Samuel Beckett est également en grande partie métalinguistique, en particulier L'Innommable (1953) ou Mal vu mal dit (1981), œuvres qui « raconte[nt] [...] entre autres histoires, celle des rapports des personnages (narrateurs ou non) avec les mots» (Clément 1994: 199) ; une grande partie de l'œuvre de Michel Leiris entre également dans cet ensemble, en particulier Langage tangage (1985) sous-titré de manière explicite "Ou ce que les mots me disent»; L'Usage de la parole (1980) de Nathalie Sarraute qui consiste à commenter des mots ou des expressions : "le mot amour ", "mon petit ", " eh bien quoi », "pourquoi pas»; Bruits de langues ${ }^{15}$ (1980) ou Le Lieu des signes de Bernard Noël sont aussi dès leur titre des œuvres métalinguistiques. On sait aussi que le projet de Francis Ponge était d'interroger les mots et leur rapport aux choses, leurs définitions et que c'est bien le mot (plus que la chose) cageot qui est «à mi-chemin de la cage au cachot » dans le poème du même nom. R. Audet (2006), dans ses travaux sur la narrativité a montré que l'événement dans un texte pouvait être un événement intramondain, « opéral » ou discursif ; dans ces œuvres, l'événement est clairement - et quasi uniquement - discursif.

Une des façons pour ce type d'œuvres de "parler des mots » est de faire des mots les personnages mêmes, de prendre à la lettre ces formules de J. Authier-Revuz soulignant que «dans tout fait de réflexivité autonymique, il y a un signe qui s'impose comme objet, propulsé sur le devant de la scène comme "personnage" auquel le dire fait référence» (2003: 71) Ainsi Ouvrez (1997) de Sarraute est un roman dont les personnages sont des mots et les chapitres des «drames » vécus par ces mots: «Des mots, des êtres vivants parfaitement autonomes, sont les protagonistes de chacun de ces drames » (p. 9). La quatrième de couverture des Premiers mots (1973) de Bernard Noël s'interroge sur l'origine énonciative du roman :

Qui parle : vous, moi, ou bien seulement des mots pressés de se reproduire? Il n'y a peut-être au monde qu'un bruit de langue. ET qu'il dise Je, ou Tu, ou Il, c'est toujours pour faire COMME SI. [...] Il faudrait apprendre à tirer la langue, mais le corps - le corps perdu ou retrouvé - en est encore à ses premiers mots.

21 B. Noël décrit La Comédie intime (2015) comme sa "petite comédie humaine », où les personnages sont des pronoms personnels, chacun des monologues qui constituent cet ouvrage interrogeant un pronom personnel qui ouvre chacune des phrases: le «je » pour La Langue d'Anna, le «tu » pour Le Mal de l'intime, le « nous » pour le Monologue du Nous, etc. Ce que soulignent ces œuvres, c'est qu'il y a un réel verbal, langagier que l'auteur comme le lecteur peut s'approprier comme expérience, et dans lequel l'auteur comme le lecteur peut se promener, pour reprendre la métaphore spatiale de M. Foucault dans Le Beau danger :

Dans cette impossibilité où je me suis trouvé d'utiliser mon propre langage, je me suis aperçu, d'abord que celui-ci avait une épaisseur, une consistance, qu'il n'était pas simplement comme l'air qu'on respire, une transparence absolument insensible, ensuite qu'il avait ses lois propres, qu'il avait ses corridors, ses chemins de facilité, ses lignes, ses pentes, ses côtes, ses aspérités, bref qu'il avait une physionomie et qu'il formait un paysage où l'on pouvait se promener et découvrir au détour des mots, autour des phrases, brusquement, des points de vue qui n'apparaissaient pas auparavant (1968 (2011): 30-31).

La fameuse définition de la littérarité comme "exploration des possibilités de la langue » reprend cette métaphore spatiale, dont Claude Simon a fait le moteur de 
plusieurs de ses romans, comme il l'explique notamment dans la préface d'Orion aveugle (Skira, 1970), lorsqu'il évoque les "mots carrefours", nœuds de signification, qui "éclatent comme autant de chandelles romaines, déployant leurs gerbes dans toutes les directions. Ils sont autant de routes où plusieurs routes s'entrecroisent ».

Dans toutes ces œuvres, le langage est thématisé ou personnifié, donc constamment rappelé, créant une opacification généralisée qui génère de l'hétérogène.

\subsection{Le discours objet du dire}

À un degré moindre, mais saturant aussi les discours, on peut distinguer des œuvres où le métalinguistique (ou méta-énonciatif) ou l'épilinguistique est au cœur du projet littéraire, du style de l'œuvre : c'est le cas des œuvres de Marivaux, Diderot, Flaubert, Jarry, Tardieu, Leiris, Queneau, Beckett, Simon, Sarraute, Barthes, Derrida, Quignard, Ernaux, Salvayre ..., autant d'auteurs dont les œuvres sont évoqués dans Ces mots qui ne vont pas de soi. Boucles réflexives et non-coïncidences du dire de Jacqueline Authier-Revuz (1995), ou dans Parler des mots (2003) publié sous la direction de J. Authier-Revuz, M. Doury et S. Reboul-Touré, et qui mettent en avant un je dis $X$ ' qui peut être traversé de comme tout le monde dit (Flaubert), comme un autre dit (Marivaux), comme certains disent (Ernaux)... Dans ces œuvres, « l'arrêt-sur-mot apparaît comme le moteur même de leur progression » (2007: 128). François Bon, dans Impatience (Minuit, 1998) souligne : «ce que nous représentons n'est pas la ville mais les mots qui la nomment» (p. 17). La notion de "discours épilinguistique " (Canut 2007), permet de rendre compte de l'ensemble de ces discours méta. Au sein des "traces épilinguistiques » que laisse l'activité épilinguistique, Cécile Canut distingue deux catégories: les gloses ou modalisations autonymiques, traces épilinguistiques au cours du dire; les discours épilinguistiques qui sont des "discours autonomes" tenus sur la langue ou les pratiques langagières (réunies sous le nom de «lecte»), qui peuvent être liés aux pratiques langagières (commentaire différé ou immédiat sur son propre discours/ langage ou sur le discours/langage d'un autre, spontané ou sollicité) ou construits à distance des pratiques (grammaires, descriptions linguistiques).

Cette mise en scène d'une parole épilinguistique et en grande partie auto-dialogique est fondamentale dans l'œuvre de Lydie Salvayre : cette activité métalinguistique passe par le dire comme objet du dire mais aussi par des formes de non-coïncidences (hétérogénéité montrée) dans La Conférence de Cintegabelle (Seuil/Verticales, 1999) notamment. Ce roman met en scène un narrateur à l'activité épilinguistique très marquée, qui crée une opacification permanente : le texte se présente comme une mise en fiction de ce je parle, par le sujet sous la forme d'une "conférence" dédiée aux bienfaits de la conversation. Les constants commentaires méta-discursifs (sur le discours en général) ou méta-énonciatifs (sur le discours en train de se faire) font de ce roman un roman saturé d'auto-dialogisme (orientation d'un énoncé « vers lui-même en tant que discours $\left.{ }^{16}\right)$. Ce discours épilinguistique repose sur un respect des normes discursives de la littérature et de la conversation classiques, une homogénéisation normative qui transparaît dans de nombreux éléments du discours, notamment les références aux auteurs de l'Antiquité au XVIII ${ }^{\mathrm{e}}$ siècle, mais aussi le style périodique :

Voici donc, chères Cintegabelloises, chers Cintegabellois, mon plan de sauvetage conçu dans la plus grande urgence et dont je n'hésite pas à dire qu'il est d'utilité nationale puisqu'en se proposant de ressusciter l'éclat de la parole aux yeux d'un monde ayant désappris de parler, il ne vise rien de moins que d'œuvrer au 
relèvement civique de notre pays et de redorer son blason afin que, je reprends mon souffle, afin que, forte du prestige reconquis, la France de demain assure de par le monde la mission civilisatrice qui lui fut, de tout temps, échue. (p. 12) subordonnées récursives, une acmée soulignée par la répétition de la locution conjonctive afin que, elle-même soulignée par une incidente je reprends mon souffle qui, tout en convoquant le corps de l'orateur, est une manière de commenter la longueur de la phrase. Un lexique et des structures phraséologiques de registre soutenu (un monde ayant désappris de parler, rien de moins que, forte du prestige reconquis, échue). L'homogénéisation normative reprenant le modèle de la période classique est en accord avec une conception politique puriste (sur la décadence de la conversation française) et colonialiste (la mission civilisatrice). Cette dernière expression comporte une forme d'opacification implicite, dans la mesure où elle est la reprise des discours de Jules Ferry et est devenue (comme expression) un symbole du colonialisme ${ }^{17}$. Mais l'extrait suit également un mouvement d'hétérogénéisation, dans le registre familier, dans les digressions sur sa vie privée, et surtout dans l'emphatisation narcissique de sa valeur de conférencier, marquée par l'autodialogisme de formules comme je n'hésite pas à dire. S'observe là une coïncidence de l'énonciateur à son dire où « l'espace du dédoublement énonciatif, distanciant, est le lieu d'une annulation emphatique, de la distance, d'un redoublement du un » (Authier-Revuz 1995(2012): 502) ; le locuteur assume pleinement son dire tout en ayant conscience de - ou en emphatisant - la dimension contestable par son auditoire de ce dire : il y a bien mise en relief de son propre dire, affiché comme remarquable, courageux. Cette mise en avant de son propre dire est aussi une manière d'exemplifier sa conception de la conversation, d'approprier son discours à l'objet de son discours, à savoir la conversation.

Vous l'avez compris, mesdames messieurs, l'homme de conversation, c'est l'artiste, l'éveillé, le solitaire, celui qui n'en appelle à aucun maître, ne se prosterne devant rien, et ne tire l'autorité de son discours que de lui-même. Il est libre, fraternel, miséricordieux, pas mesquin pour deux sous, quoi encore ?, de l'esprit à revendre, épris de beauté, très sympathique, et tout à l'avenant. L'homme de la conversation, en un mot, c'est moi. Je ne puis vous le cacher plus longtemps.

Comment m'y prends-je? Je vends la mèche. Profitez-en. Voilà. Je cueille une opinion ici, dans la boucherie de $\mathrm{M}$. Tribulet, en intercepte une autre là, dans le journal, dérobe un argument à Sénèque, médite une phrase de Saint-Simon, puis examine ma récolte, assemble les parties, les désassemble, les recompose jusqu'à les faire miennes, jusqu'à les faire chair. (p. 85)

Dans cet énoncé, le narrateur réussit à joindre la revendication d'un discours à soi et la conscience du dialogisme interdiscursif; l'opacification est bien généralisée : sensible tout d'abord dans l'interdiscours homogénéisant des normes de la langue classique et du genre de discours très contrôlé qu'est la "conférence ", elle apparaît également dans le dialogisme interlocutif du rapport à son auditoire et dans l'auto-dialogisme emphatique d'un "discours à soi », formes d'hétérogénéité par rapport aux normes discursives.

\subsection{Genres hétérogènes ou homogènes}

Le métalinguistique et l'hétérogénéité peuvent aussi être observés à un niveau plus large, qui n'est plus celui de telle œuvre prenant le langage ou le discours comme objets du dire, mais celui du genre même, en soi hétérogène ou homogène. Ainsi pour 
Bakhtine, alors que le roman représente un acte d'énonciation, «le poème est un acte d'énonciation " (Todorov 1981: 101); il réserve l'hétérogénéité au genre romanesque dans Esthétique et théorie du roman :

Dans la représentation poétique au sens propre du terme [...], toute l'action [...] se joue entre le mot et l'objet (sous tous leurs aspects). Le mot se coule dans la richesse inépuisable, dans la multiformité contradictoire de l'objet lui-même [...]. C'est pourquoi il ne présuppose rien au-delà des limites de son contexte sinon, s'entend, les trésors du langage lui-même. [...]

Pour l'artiste-prosateur, au contraire, l'objet révèle avant tout la multiformité sociale, plurilingue de ses noms, définitions et appréciations. Au lieu de la plénitude inépuisable de l'objet lui-même, le prosateur découvre une multitude de chemins, routes, sentiers, tracés en lui par sa conscience sociale. (1978: 101-102)

Cette réunion d'unités stylistiques hétérogènes - et en grande partie métalinguistiques - permettrait de caractériser le genre du roman: "L'originalité stylistique du genre romanesque réside dans l'assemblage de ces unités dépendantes, mais relativement autonomes» (p. 88); «Le roman c'est la diversité sociale de langages, parfois de langues et de voix individuelles, diversité littérairement organisée » (p. 88). Ce plurilinguisme qui spécifie le discours romanesque fait donc du dialogisme un élément de la valeur de l'œuvre: "L'orientation dialogique du discours parmi les discours "étrangers" (à divers degrés et de diverses manières) lui crée des possibilités littéraires neuves et substantielles, lui donne l'artisticité de sa prose.» (p. 99).

Face au genre romanesque, le genre poétique serait, à l'inverse, monolingue : «l'unité du système du langage, l'unité (et l'unicité) de l'individualité linguistique et verbale du poète, sont le postulat indispensable du style poétique » (p. 90) :

Le langage du poète, c'est son langage à lui. Il s'y trouve tout entier, sans partage. Il utilise chaque forme, chaque mot, chaque expression dans leur sens direct («sans guillemets ", pour ainsi dire). (p. 108)

31 Si l'on suit Bakhtine donc, la poésie mettrait en jeu des formes d'hétérogénéités énonciatives non-dialogiques, relevant des non-coïncidences entre les mots et les choses et des mots à eux-mêmes, alors que le roman convoquerait davantage les noncoïncidences dialogiques (interlocutives et interdiscursives), même si Bakhtine nuance faisant référence à la poésie d'Horace, de Villon, d'Heine, de Laforgue et d'Annenski : « Il est vrai qu'une image dialogisée de cet ordre peut trouver sa place (sans donner le ton) dans tous les genres poétiques, même dans la poésie » (p. 101). On pourrait ajouter à cette liste, parmi de nombreux autres exemples (Cendrars, Desnos, Queneau, etc.), les «poèmes-conversations » d'Apollinaire («Lundi rue Christine »), ou plus récemment Ceci est un poème qui guérit les poissons de Jean-Pierre Siméon et Olivier Tallec (Rue du monde, 2005), poèmes construits à partir de bribes de discours autres. De tels textes soulignent la proximité entre la langue commune, ordinaire, parcourue par de l'hétérogène manifeste et le discours littéraire où l'hétérogène est constamment manifesté et représenté. Hétérogènes par leur genre (la conversation quotidienne) intégré à un genre de discours second (le roman), ces genres de discours premiers perdent leur transparence relative ou apparente, s'opacifient par cette intégration :

$\mathrm{Au}$ cours du processus de leur formation, ces genres seconds absorbent et transmutent les genres premiers (simples) de toutes sortes, qui se sont constitués dans les circonstances d'un échange verbal spontané. Les genres premiers, en devenant composantes des genres seconds, s'y transforment et se dotent d'une caractéristique particulière : ils perdent leur rapport immédiat au réel existant et au réel des énoncés d'autrui - insérée dans un roman, par exemple, la réplique $d u$ 
dialogue quotidien ou la lettre, tout en conservant sa forme et sa signification quotidienne sur le plan du seul contenu du roman, ne s'intègre au réel existant qu'à travers le roman pris comme un tout, c'est-à-dire le roman conçu comme phénomène de la vie littéraire-artistique et non de la vie quotidienne ${ }^{18}$.

L'intégration à une œuvre opacifie donc forcément, crée ce que C. Rannoux nomme (icimême) un effet métalinguistique qui s'applique ici à l'œuvre entière. On retrouve cette opacification générique dans le travail de Jean-Charles Massera et sa reprise des genres de discours publicitaires, juridiques, médiatiques... (voir Bikialo 2014 et 2017), avec en arrière-plan l'idée que «l'opaque est aussi une arme critique poétique et/ou politique. Un des outils de l'insurrection quotidienne» (Gleize 2011: 44).

\section{Conclusion}

Nous campons près d'Ajaccio. Il fait très beau. On mange bien. J'ai pris un coup de soleil. Bons baisers.

On conviendra qu'un tel texte n'est ni métalinguistique ni hétérogène. Sauf lorsqu'il constitue la première des «Deux cent quarante-trois cartes postales en couleurs véritables » de Georges Perec ${ }^{19}$ : par cette insertion dans une œuvre, dans un projet d'écriture faisant place aux clichés interdiscursifs des cartes postales estivales, le texte est comme autonymisé ( $"$ je dis $X^{\prime}$ ou $X^{\prime}$ = comme on dit dans une carte postale). La littérarité (au sens d'ensemble de traits formels qui feraient d'une œuvre une œuvre littéraire) n'existe pas : mais la valeur du discours littéraire s'évalue en partie par la manière dont les formes de non-coïncidence (les comme on dit, comme il dit) se résolvent donc en un je dis $X^{\prime}$ au sein d'une œuvre. Si cette dimension métalinguistique et hétérogène de l'œuvre est parfois représentée ou manifeste, elle est aussi, d'un point de vue théorique, constituante. C'est souvent le lecteur - et plus encore le lecteur critique ou le critique linguiste - qui va percevoir, voire interpréter cette hétérogénéité et/ou cette dimension métalinguistique, hétérogénéité et méta- interprétatifs et aléatoires donc, non nécessaires à la compréhension mais nécessaires au sens.

\section{BIBLIOGRAPHIE}

Adam J.-M. (2012). Arrêt-sur-mot et narrativisation d'un concept : le "pénitentialisme" de la CIA et les Trois petits cochons. In S. Branca-Rosoff, C. Doquet, J. Lefebvre, E. Oppermann-Marsaux, S. Pétillon et F. Sitri (eds), L'Hétérogène à l'œuvre dans la langue et les discours. Hommage à Jacqueline Authier-Revuz. Limoges : Lambert-Lucas. 59-68.

Aquien M. (1989). L'Autre versant du langage. Paris : Corti.

Aron T. (1984). Littérature et littérarité. Un essai de mise au point. Annales littéraires de l'Université de Besançon, Les Belles lettres.

Audet R. (2006). La narrativité est affaire d'événement. In D. Rivière (éd.), Jeux et enjeux de la narrativité dans les pratiques contemporaines. Paris : Éditions Dis voir (Arts visuels/essais). 7-35. 
Authier-Revuz J. (1984). Hétérogénéité(s) énonciative(s). Langages 73. 98-111.

Authier-Revuz J. (1995(2012)). Ces mots qui ne vont pas de soi. Boucles réflexives et non-coïncidences du dire. Limoges : Lambert Lucas.

Authier-Revuz J. (2000). Aux risques de l'allusion. In M. Murat (éd.), L'Allusion dans la littérature. Paris : Presses Universitaires Paris Sorbonne.

Authier-Revuz J. (2003). Le fait autonymique : Langage, langue, discours. Quelques repères. In J. Authier-Revuz, M. Doury et S. Reboul-Touré (eds), Parler des mots. Le fait autonymique en discours. Paris : Presses Sorbonne Nouvelle.

Authier-Revuz J. (2007). Arrêts sur mots. L'épreuve de la langue dans l'énonciation et l'écriture. In I. Fénoglio (éd.), L'Ecriture et le souci de la langue. Louvain-La-Neuve : Bruylant Academia. 113-145.

Bakhtine M. (1934-1935 (1978)). Esthétique et théorie du roman. Paris : Gallimard, coll. « Tel ». Bakhtine M. (1953 (1984)). Esthétique de la création verbale, trad. fr. : Gallimard.

Bikialo S. (2012). Coïncidences du dire : Jacqueline Authier-Revuz et Bernard Noël. In S. BrancaRosoff, C. Doquet, J. Lefebvre, E. Oppermann-Marsaux, S. Pétillon et F. Sitri (eds), L'Hétérogène à l'œuvre dans la langue et les discours. Hommage à Jacqueline Authier-Revuz. Limoges : LambertLucas. 337-347.

Bikialo S. (2014). Genres de discours et réalité dans la fiction narrative contemporaine. In C. Narjoux et C. Stolz (eds), Fictions narratives du XXI ${ }^{e}$ siècle : approches stylistiques, rhétoriques, sémiotiques. La Licorne 114. Rennes : PUR. 85-99.

Bikialo S. et Rannoux C. (2017). Naissance d'un pont : une "chorégraphie de la collision". In M. Bonazzi, C. Narjoux et I. Serça (eds), Maylis de Kérangal, une écriture nomade. Editions Universitaires de Dijon. 143-160

Bikialo S. (2019). Discours hors-normes et discours littéraire : ce qu'en disent la ponctuation et les genres de discours. In K. Colette et A. Steuckardt (eds), Écrits hors-normes. Université de Sherbrooke. EDUS, en ligne : https://savoirs.usherbrooke.ca/handle/11143/15583.

Bordas É. (2012). La stylistique et la question de la valeur : l'enjeu théorique. In S. Bikialo et S. Pétillon (eds), Dans l'atelier du style. Du manuscrit à l'œuvre publiée. La Licorne 98. Rennes : PUR. 79-89.

Branca-Rosoff S. (2007). Normes et genres de discours. Le cas des émissions de libre antenne sur les radios jeunes. Langage et société 119. 111-128.

Bres J. et Nowakowska A. (2005). Dis-moi avec qui tu "dialogues", je te dirai qui tu es... De la pertinence de la notion de dialogisme pour l'analyse du discours. Marges linguistiques 9. 137-153.

Canut C. (2000). Subjectivité, imaginaires et fantasmes des langues : la mise en discours "épilinguistique". Langage et société 93. 71-97.

Canut C. (2007). L'épilinguistique en question. In G. Siouffi et A. Steuckardt (eds.), Les Linguistes et la norme. Berne : Peter Lang. 49-72.

Clément B. (1994). L'Eeuvre sans qualités : rhétorique de Samuel Beckett. Paris : Seuil.

Dumarsais (1730 (1988)), Des tropes ou des différents sens (présentation, note et traduction de F. Douay-Soublin). Paris : Flammarion.

Foucault M. (1966a). La pensée du dehors. Critique 229. In Dits et écrits I, 1954-1975. Paris :

Gallimard, « Quarto ». 546-567. 
Foucault M. (1966b). Les Mots et les choses. Paris : Gallimard, « Tel ».

Foucault M. (1968 (2011)). Le Beau danger. Entretiens avec Claude Bonnefoy. Paris : Éditions de l'EHESS.

Gleize J.-M. (2011). Opacité critique. In « Toi aussi, tu as des armes... ». La Fabrique.

Lakoff G. et Johnson M. (1980 (1985)). Les Métaphores dans la vie quotidienne, trad. fr. : Minuit.

Lavis G. (1972). A propos de l'interprétation autonymique de la littérarité. Cahiers d'analyse textuelle 14. 50-66.

Lavis G. (1975). Connotation autonymique et littérarité (réponse à J. Rey-Debove). Cahiers d'analyse textuelle 17. 110-128.

Philippe G. (2002). Sujet, verbe, complément : Le moment grammatical de la littérature française 1890-1940. Paris : Gallimard.

Rabaté D. (1991). Vers une littérature de l'épuisement. Paris : Corti.

Rannoux C. (2010). La "langue de tous", un défi à la "langue littéraire", Les Années d'Annie Ernaux. In C. Narjoux (éd.), La Langue littéraire à l'aube du XXI ème siècle. Presses Universitaires de Dijon.

175-186.

Rey-Debove J. (1971). Notes sur une interprétation autonymique de la littérarité : le mode du "comme je dis". Littérature 4. 90-95.

Rey-Debove J. (1974). Autonymie et sémiotique littéraire. Littérature 16. 107-116.

Rey-Debove J. (1978 (1997)). Le Métalangage. Paris : Armand Colin.

Todorov T. (1981). Mikhail Bakhtine le principe dialogique. Paris : Seuil.

\section{NOTES}

1. Rappelons qu'on désigne par littérarité, depuis Jakobson, ce qui formerait une «spécificité du texte littéraire ». Voir la synthèse de T. Aron (1984). Autant la valeur d'un discours littéraire me semble un enjeu pertinent et encore à défricher (voir Bordas 2012), autant la littérarité, ou l'idée d'une «langue littéraire ", est à penser "non pas en termes de réalités langagières, dont la saisie s'avère des plus aléatoires, mais bien en termes d'imaginaire linguistique » (Rannoux 2010: 176). Un imaginaire linguistique qui provoque encore des réactions enflammées comme en ont témoigné, en octobre 2016, certaines réactions horrifiées à la remise du Prix Nobel de littérature à Bob Dylan.

2. S. Branca-Rosoff (2007: 117-118) écrit ainsi que «les genres littéraires voient s'équilibrer un principe de conformité qui les inscrit dans une tradition et un principe d'originalité qui nécessite que chaque œuvre fasse un écart par rapport aux œuvres déjà existantes dans le champ littéraire. La valeur littéraire est au prix de ce renouvellement. ». Voir S. Bikialo (2019) pour des éléments sur le lien entre surprise, nouveauté, originalité et hors normes aux niveaux des genres de discours et de la ponctuation.

3. J. Authier-Revuz (1984: 99) définit l'hétérogène montré de la façon suivante: «formes linguistiques représentent des modes divers de négociation du sujet parlant avec l'hétérogénéité constitutive de son discours"

4. Me semble aller dans ce sens cette remarque de J.-M. Adam (2012: 59) : « C'est souvent dans le repli d'une non-coïncidence manifeste [...] que se fait le sens, que se déploie une idéologie, et sous les mots, une densité de langage, un excès de signifiance, une ouverture du discours sur son interdiscursivité constitutive. » 
5. Au sens défini par C. Canut (2007: 50-51) : "J'ai repris [...] le terme épilinguistique pour montrer que l'activité épilinguistique dégagée par Culioli peut entraîner toutefois des discours autonomes sur les formes langagières par tous les locuteurs (y compris les linguistes), nous autorisant à concevoir les discours épilinguistiques comme une catégorie recouvrant aussi les discours métalinguistiques, quelle que soit leur objectivation scientifique. Ils caractérisent donc tout type de discours autonome sur les langues ou les pratiques. Ces discours ne peuvent, en euxmêmes, nous dire tout du langage puisque, fondamentalement, c'est dans cette part qui échappe au chercheur que se loge la relation singulière du sujet au langage faite d'abord de sentiments, d'impressions et d'imaginaires. "

6. Rabaté (1991: 190-191). Il indique aussi : "c'est un mixte indissociable de fictions et de spéculations théoriques. Un espace où jouent des savoirs pris dans une énonciation qui les met à distance, les donne à entendre comme autant de fables. » (p. 46).

7. «On appelle connotation langagière réflexive, ou autonymique, la situation d'un signe qui signifie, comme connotateur, son signifiant et son signifié dénotatif [...]. La connotation autonymique cumule deux sémiotiques : on emploie un signe et on le cite tout à la fois. » (ReyDebove 1978(1997): 253-254)

8. C'est en 1971, dans un article paru dans la revue Littérature en 1971 et intitulé « Notes sur une interprétation autonymique de la littérarité : le mode du "comme je dis" » que J. Rey-Debove fait cette proposition, qui donnera lieu à un débat par article interposés, avec G. Lavis $(1972,1975)$; l'article est repris dans Le Métalangage (1978(1997): 286-291).

9. Ce qui est lié aux différentes formes de « redondance » (1971: 93 et 1978(1997): 289).

10. C'est ainsi que Jakobson (1963: 220) définit la fonction poétique : « La fonction poétique projette le principe d'équivalence de l'axe de la sélection sur l'axe de la combinaison. L'équivalence est promue au rang de procédé constitutif de la séquence. » Aron (1984: 37-41) développe les enjeux et conséquences de ce principe d'équivalence.

11. S. Bikialo et C. Rannoux (2017) analysent cette condensation et densité au sein d'une œuvre (Naissance d'un pont de Maylis de Kérangal).

12. J. Authier-Revuz (2007: 113). Dans « Aux risques de l'allusion» (2000), J. Authier-Revuz aborde quelques-uns de ces risques chez Flaubert et Mallarmé.

13. Même si cela dépend à coup sûr des discours, et qu'on peut encore constater comme Dumarsais en 1730 qu'« il se fait plus de figures en un seul jour de marché à la halle qu'il ne s'en fait en plusieurs jours d'assemblées académiques. Ainsi, bien loin que les figures s'éloignent du langage ordinaire des hommes, ce serait au contraire les façons de parler sans figures, qui s'en éloigneraient, s'il était possible de faire un discours où il n'y eût que des expressions non figurées. » (1730(1988): 62-63). Dans Les Métaphores dans la vie quotidienne, G. Lakoff et M. Johnson (1980) soulignent eux aussi à leur manière que la métaphore n'est pas un trait possible du discours littéraire étant donnée son omniprésence (présence et densité) dans le discours ordinaire.

14. P. Fournel l'explicite dans une vidéo présente sur le site de son éditeur: http://www.polediteur.com/index.php?spec=videos-sons\&numpage=20\&numrub=11\&numlivre=6466, $\quad 5 \mathrm{mn}$. Consulté le 20 février 2017.

15. Voir Bikialo (2012).

16. Bres et Nowakowska (2005: 139).

17. En référence au discours sur la colonisation de Jules Ferry en juillet 1885 ( « Il y a un second point que je dois aborder... : c'est le côté humanitaire et civilisateur de la question... Les races supérieures ont un droit vis-à-vis des races inférieures. Je dis qu'il y a pour elles un droit parce qu'il y a un devoir pour elles. Elles ont le devoir de civiliser les races inférieures. »), on peut citer notamment l'ouvrage de Dino Costantini, Mission civilisatrice. Le rôle de l'histoire coloniale dans la construction de l'identité politique française, La Découverte, 2008. 
18. M. Bakhtine (1952-1953(1984): 267). J'aborde ces enjeux avec de nombreux exemples dans la littérature contemporaine en prose dans (Bikialo 2014).

19. G. Perec, « Deux cent quarante-trois cartes postales en couleurs véritables », dans Le Fou parle n 8, octobre 1978, repris dans L'Infra-ordinaire, Seuil, 1989, p. 33.

\section{RÉSUMÉS}

Cet article s'interroge sur la place et la fonction du métalinguistique et de l'hétérogénéité dans la valeur du discours littéraire. À partir de l'hypothèse émise par J. Rey-Debove sur la littérature comme mode du comme je dis, reformulée, à la lumière des travaux de J. Authier-Revuz, en mode $\mathrm{du} J e$ dis $X^{\prime}$ (où $X^{\prime}$ est un signe en modalisation autonymique), l'article évoque des œuvres (contemporaines) où le langage est "rappelé » avant de s'attacher aux genres de discours littéraires (roman et poésie) et à leur hétérogénéité respective en lien avec les genres de discours quotidiens.

This article examines the place and function of the metalinguistic and heterogeneity in the value of literary discourse. From the hypothesis by J. Rey-Debove on literature as mode of as I say, reformulated in light of the work of J. Authier-Revuz, in the way I say $X^{\prime}$ (where $X^{\prime}$ is a sign modalisation autonymique), the article evokes (contemporary) works where language is "reminded" before attaching the literary discourse genres (novel and poetry) and their respective heterogeneity in dregs with the genres of daily speeches.

\section{INDEX}

Mots-clés : Métalinguistique, littérarité, valeur, hétérogénéité, opacification, mode de dire Keywords : Metalinguistic, literarity, value, opacification, way of saying

\section{AUTEUR}

\section{STÉPHANE BIKIALO}

Université de Poitiers, FoReLLIS 